\title{
Introducción
}

Chile, después de tener la mayor mortalidad por tuberculosis de América durante la primera mitad del siglo $\mathrm{XX}$, ha pasado a tener durante la segunda mitad de ese siglo, una de las tasas de incidencia de la enfermedad más bajas de la región. Esto se ha debido, esencialmente, a la implementación de un moderno Programa de Control de la Tuberculosis. Es así como Chile pertenece al selecto grupo de "países de baja prevalencia de tuberculosis de las Américas" desde hace 14 años.

Durante la VI Reunión de este grupo en Santiago, en abril de este año, se analizaron las dificultades por las que atraviesan estos países en su tránsito hacia la eliminación de la tuberculosis y el menor descenso de la incidencia de la enfermedad que se está observando en los últimos años. Como ha sido señalado reiteradamente, este deterioro en la situación epidemiológica de la tuberculosis frecuentemente corre paralelo con la reducción de los recursos asignados a su control.

En este número de la revista la Dra. Tania Herrera, Directora del Programa Nacional de Control de la Tuberculosis del Ministerio de Salud hace un resumen de los resultados de esta reunión y el Dr. Álvaro Yáñez, ex Asesor Regional de Tuberculosis de la Organización Panamericana de la Salud, hace un interesante análisis sobre la disminución del interés y apoyo que está experimentando el control de la tuberculosis por parte de autoridades, profesionales y de la población en general, a medida que la enfermedad se hace menos frecuente y, erradamente, se estima por muchos que desaparecerá por sí sola.

\section{Reunión de países de baja prevalencia de tuberculosis en Las Américas}

\author{
TANIA HERRERA M.*
}

\section{$6^{\text {th }}$ Meeting of low prevalence countries of tuberculosis in Latin America and the Caribbean}

In April of 2013, the sixth version of the Meeting of Low Prevalence Countries (LPC) of tuberculosis in Latin America and the Caribbean was carried out in Santiago de Chile, which was attended by the heads of National Program of Tuberculosis of Uruguay, Costa Rica, Cuba and Chile. These countries discussed the epidemiological situation and the operational results of the implementation of strategies for the elimination of tuberculosis, which involves reaching an incidence rate of less than 1 case per million inhabitants in 2050. During the last decade, LPC have experienced a slowing of the decline in the incidence of tuberculosis that even resulted in an epidemiological reversal in Uruguay, which makes unlikely the possibility of achieving the goal. Moreover, the operational situation varies between countries, showing the biggest problems in Uruguay and Chile, who do not meet the goals in treatment cohorts. The political commitment of the authorities and the adequate financing of the National Tuberculosis Programme, along with the inter-sector coordination, were highlighted as key elements to meet the challenges that it has the control of tuberculosis in the LPC.

Key words: Tuberculosis, control, prevalence, meeting.

\section{Resumen}

En abril de 2013 se realizó en Santiago de Chile la VI versión de la Reunión de Países de baja prevalencia (PBP) de tuberculosis de Latinoamérica y el Caribe, a la cual asistieron los jefes de programa de Uruguay, Costa Rica, Cuba y Chile. Los países discutieron la situación epidemiológica y los

* Médico, Directora del Programa Nacional de Control de la Tuberculosis. División de Prevención y Control de Enfermedades Ministerio de Salud. Chile. 
resultados operacionales de la implementación de estrategias para la eliminación de la tuberculosis al año 2050, lo que implica llegar a una tasa de incidencia menor a 1 caso por millón de habitantes. Durante la última década, los PBP han experimentado un enlentecimiento de la disminución de la incidencia de tuberculosis que incluso ha resultado en una reversión epidemiológica para Uruguay, lo que aleja la posibilidad de alcanzar las metas establecidas. Por otra parte, la situación operacional es variable entre los países, evidenciándose los mayores problemas en Uruguay y Chile, quienes no cumplen con las metas en las cohortes de tratamiento. El compromiso político de las autoridades y el adecuado financiamiento de los Programas Nacionales de Tuberculosis, junto con la coordinación intersectorial, fueron destacados como los elementos claves para enfrentar los desafios que tiene el control de la tuberculosis en los PBP.

Palabras clave: Tuberculosis, control, prevalencia, reunión.

\section{Introducción}

A partir de 1999, la Organización Panamericana de la Salud ha promovido y auspiciado la Reunión Subregional de Países de Baja Prevalencia de Tuberculosis de las Américas. La primera reunión fue realizada ese año en San José de Costa Rica con la participación de Chile, Cuba, Uruguay y Venezuela como observador, y en ella se analizó la situación de la tuberculosis (TB) y se definieron los indicadores y etapas de la eliminación, junto con estrategias de intervención especiales. Así, se estableció que la meta de eliminación de la TB como problema de Salud Pública correspondería a una tasa de incidencia menor a 5 casos por 100.000 habitantes.

Las siguientes reuniones se desarrollaron en Uruguay (2001), Chile (2004), Costa Rica (2006) y Puerto Rico (2011). En estas reuniones se abordaron los temas de viabilidad y amenazas para las estrategias de eliminación, la estratificación de grupos de riesgo y uso de sistemas de registro, la implementación de intervenciones relacionadas con la co-infección TB-VIH y manejo de la TB Multidrogorresistente (resistente a rifampcicina e isoniacida: TB-MDR) y, durante la reunión en Puerto Rico el año 2011 se definió que la eliminación de la TB corresponde a la reducción de la incidencia a menos de 1 caso por un millón de habitantes por año.

La sexta versión de la Reunión de Países de Baja Prevalencia (PBP) de Tuberculosis de las Américas se llevó a cabo en Santiago de Chile entre los días 16 y 18 de abril del presente año. Asistieron al encuentro los encargados nacionales de los Programas de Tuberculosis de América Latina y el Caribe: Cuba, Costa Rica, Uruguay y Chile, además de expertos de los Programas de TB de Estados Unidos, Canadá, Holanda y de la OMS, convocados por la Oficina Regional para el Control de la Tuberculosis de la OPS.

Los objetivos planteados para la reunión fueron:
- Discutir las perspectivas de alcanzar las metas de eliminación definidas en la $\mathrm{V}$ reunión de PBP el año 2011.

- Revisar e identificar los indicadores para definir las metas programáticas de eliminación en los próximos años.

- Conocer la situación de implementación de los planes de eliminación en los PBP.

- Discutir estrategias para la atención integral de salud y el trabajo inter-programático para combatir los determinantes sociales de salud en población vulnerable.

- Compartir estrategias de eliminación entre los países.

La metodología de trabajo de la reunión consistió en presentaciones de la situación de avance hacia la eliminación de la tuberculosis de los PBP de Latinoamérica y el Caribe, el rol de los laboratorios en el proceso de eliminación de la tuberculosis y el trabajo que se ha realizado con poblaciones vulnerables en estos países. Por otra parte, se realizaron presentaciones de los expertos de Estados Unidos, Canadá y Holanda, países cuyas tasas de incidencia ya están por debajo de la etapa de eliminación avanzada de la tuberculosis (tasa de incidencia de 5 por 100.000 habitantes), quienes plantearon los nuevos desafíos a los que se enfrentan los países que se encaminan a la eliminación de la enfermedad (tasa de incidencia menor a 1 por un millón de habitantes). Además de las presentaciones, se realizaron talleres grupales para definir los principales componentes de los planes de eliminación de los países y los indicadores y metas más importantes para monitorizar y evaluar estos planes.

\section{Progresos hacia la eliminación de la Tuberculosis}

Las presentaciones de la situación de la tuberculosis en los cuatro países de baja prevalencia de Latinoamérica y el Caribe, dan cuenta de un 
enlentecimiento en la reducción de las tasas de incidencia de la tuberculosis en todas las formas (TBTF) que incluye casos nuevos y recaídas, mostrando incluso aumentos en 2011 que corresponde al último año notificado (Figura 1). La situación más preocupante la presenta Uruguay, país que luego de haber alcanzado una tasa de incidencia notificada de 16,7 el año 2006 , ha presentado un progresivo aumento de la incidencia de TB llegando a 24,2 el año 2011. Más del 50\% de los casos en Uruguay se producen en Montevideo, cuya tasa de incidencia es de 35,5 por 100.000 habitantes, siendo los grupos de mayor riesgo los usuarios de drogas duras y las personas con VIH.

En Cuba, luego de un repunte producido a partir de 1992 y que requirió de un reforzamiento del Programa de Tuberculosis para controlar esta reversión epidemiológica, ha mantenido tasas de incidencia de TBTF entre 6 y 8 por 100.000 habitantes durante la última década, con una tasa de incidencia notificada de 7,2 para el año 2011, la más baja de los cuatro PBP, pero similar a la tasa del año anterior. Los grupos identificados como de mayor riesgo en Cuba son los alcohólicos y los adultos mayores.

En el caso de Costa Rica, la incidencia de TBTF venía disminuyendo en forma lenta hasta el 2009, cuando alcanzó una tasa de 9,6 por 100.000 , pero en los últimos dos años se ha producido un leve aumento hasta una tasa de 10,9 el 2011. El principal riesgo identificado es la pobreza, especialmente cuando se asocia a poblaciones privadas de libertad, migrantes provenientes de Nicaragua o poblaciones indígenas.

La incidencia de TBTF en Chile también ha venido disminuyendo en forma muy lenta durante la última década, con un leve aumento el año 2011 cuando alcanzó una tasa de 14,2 por 100.000 habitantes (Figura 1). Los principales grupos de riesgo identificados en Chile son las personas con VIH, privadas de libertad y los extranjeros provenientes de países de alta endemia, además de aquellas personas que viven en zonas de mayor pobreza.

El porcentaje de coinfectados TB-VIH del total de casos notificados con TBTF en el año 2011 corresponde a un 14,2\% para Uruguay, 9,2\% para Cuba, $8 \%$ para Costa Rica y $6 \%$ para Chile. En cuanto a la situación de la Tuberculosis Multidrogorresistente (TB-MDR), todos los países han mantenido pocos casos, así según datos del Global Report 2012 de la OMS, para el año 2011 Chile presentó 15 casos, Costa Rica 3 casos, Cuba 4 casos, y Uruguay 2 casos.

Las esperanzas de alcanzar las metas de eliminación de la tuberculosis para el año 2050 se alejan a la luz de estos resultados. Uruguay requeriría de una disminución de un $13 \%$ anual en la incidencia de TBTF para poder llegar a eliminar la enfermedad, en tanto que en la década 2001 a 2011 esta disminución fue de sólo un $1,3 \%$. Chile tuvo un 3\% de reducción anual para esta misma década, y requeriría una disminución de un $11 \%$ anual para la eliminación de la TB el año 2050. Cuba tuvo un 2,3\% de reducción anual y requeriría una reducción de $10 \%$ anual para alcanzar la meta al 2050, y Costa Rica disminuyó a una velocidad de $6 \%$, pero requeriría que esta cifra fuese de un $11 \%$ para eliminar la TB.

\section{Situación operacional de los países de baja prevalencia}

Se espera que los PBP logren localizar al 90\% de los casos de TB estimados por la OMS para

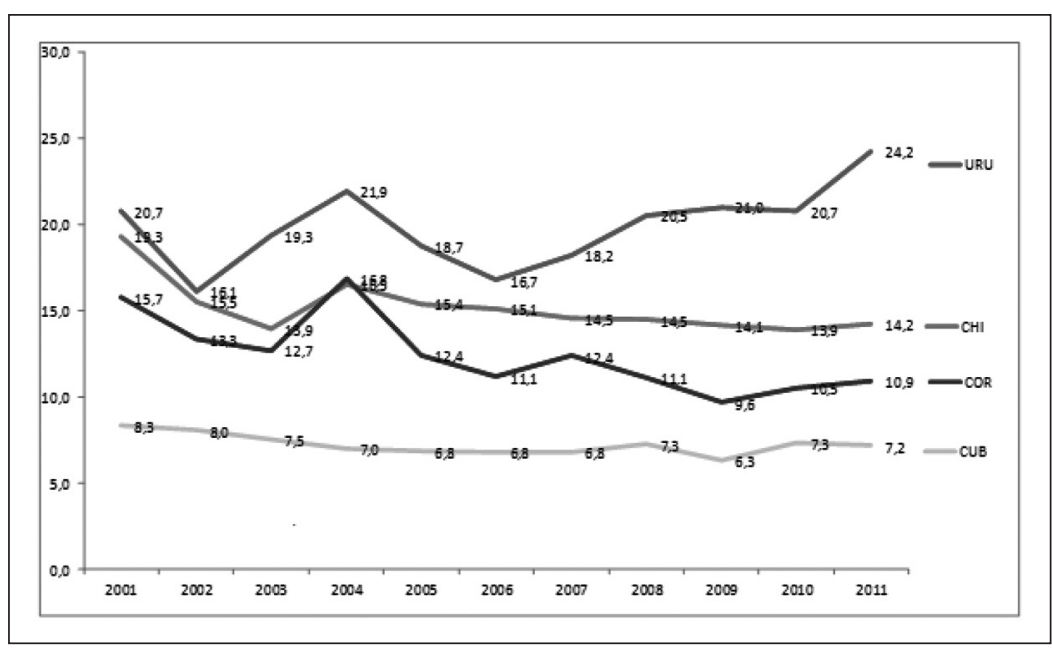

Figura 1. Incidencia notificada de Tuberculosis todas las formas 2001-2011 en los 4 países de baja prevalencia de tuberculosis de América Latina y el Caribe. Los valores numéricos de la ordenada y de las curvas representan las tasas de incidencia por 100.000 habitantes. CUB: Cuba; COR: Costa Rica; CHI: Chile; URU: Uruguay. 
cumplir con la meta operacional de la localización de casos. Para el año 2011, con excepción de Uruguay, los PBP no lograron cumplir con esta meta, siendo este porcentaje de $89 \%$ para Costa Rica, $81 \%$ para Cuba, y $79 \%$ para Chile. Uruguay presentó una localización de casos de $113 \%$ debido al aumento de casos que se ha producido en este país, por lo que se deberán hacer ajustes al cálculo de las tasas estimadas para este país.

En relación a los resultados del tratamiento, existen dificultades para la comparación de las cohortes debido a que la metodología que se ha usado varía de un país a otro, especialmente en lo que se refiere a quiénes se incluyen en la cohorte. Los porcentajes de alta de tratamiento para la cohorte de 2011 (curación confirmada o término de tratamiento) que presentaron los PBP en esta reunión son los siguientes: Uruguay $83,6 \%$, Cuba $88 \%$, Costa Rica $88 \%$ y Chile $82,1 \%$. Por lo tanto, Uruguay y Chile no están cumpliendo con la meta de OMS de alcanzar un porcentaje de éxito de tratamiento de por lo menos un $85 \%$.

Los abandonos y los fallecidos son los dos componentes que merman las cohortes. El mayor porcentaje de fallecidos lo presenta Uruguay con un $9,2 \%$, seguido de Chile con $8,5 \%$, Cuba con $7,6 \%$ y Costa Rica con 7\%. En el caso de los abandonos, Uruguay presenta un $8,3 \%$, Chile $6,5 \%$, Cuba $2,6 \%$ y Costa Rica $2 \%$.

Con respecto a las actividades de colaboración para la coinfección TB-VIH, el porcentaje de casos de tuberculosis a quienes se les realizó la prueba para detectar VIH es de $95 \%$ en Cuba y Costa Rica, $91 \%$ en Uruguay y sólo $10 \%$ en Chile. El éxito de tratamiento de la cohorte de casos nuevos de TB-VIH para el año 2010 es de $85 \%$ para Costa Rica, $70 \%$ para Cuba y $60 \%$ para Uruguay, con porcentajes de fallecidos de $13 \%$, $16 \%$ y $30 \%$ respectivamente. Para Chile, esta información no está disponible.

Finalmente, respecto a la vigilancia de la resistencia a fármacos, el porcentaje de casos nuevos de tuberculosis pulmonar a quienes se les realizó Prueba de Susceptibilidad a fármacos fue de 75\% en Uruguay, $60 \%$ en Cuba, 9,6\% en Costa Rica y $4,8 \%$ en Chile. Cabe señalar que existen diferencias en la normativa de los programas en relación a la vigilancia de resistencia en los casos nuevos. En el caso de los antes tratados, el porcentaje de casos con tuberculosis pulmonar a quienes se les realizó prueba de susceptibilidad a fármacos fue de $100 \%$ en Chile y Cuba, $72 \%$ en Uruguay y $62 \%$ en Costa Rica.

Al discutirse en los talleres grupales los indicadores que se están usando para evaluar la situación epidemiológica y operacional de los
Programas de Tuberculosis, los PBP concuerdan con que los indicadores y metas propuestas deben ser mantenidos y que son los Programas Nacionales los que deben realizar los esfuerzos para alcanzar estas metas. Los cuatro países concordaron en que la implementación de planes de eliminación requiere del compromiso político de las autoridades que se manifieste en la disponibilidad de los recursos humanos, materiales y financieros que son necesarios para avanzar hacia la eliminación de la tuberculosis, especialmente en lo que se refiere a mejorar la localización de los casos reduciendo los tiempos de diagnóstico a través de la implementación de nuevas tecnologías, y la necesidad de desarrollar estrategias intersectoriales para combatir los determinantes sociales de salud en las poblaciones vulnerables.

\section{Conclusiones y acuerdos de la reunión}

Luego de las presentaciones y discusiones en torno a la situación de la tuberculosis en los PBP, los representantes de estos países y la OPS se comprometieron a:

- Fortalecer la implementación de las actividades del estudio de contactos y acordar procedimientos para el uso de terapia preventiva definiendo los grupos priorizados.

- Mantener el registro nominal de casos de tuberculosis incorporando variables sociodemográficas, de modo de desarrollar estrategias focalizadas en grupos de riesgo.

- Definir el nivel de implementación y los algoritmos de utilización para tecnologías de biología molecular para el diagnóstico precoz de tuberculosis (Xpert MTB/RIF).

- Fortalecer las actividades de colaboración con VIH y coordinar actividades con el Programa de diabetes mellitus.

- Introducir estrategias comunicacionales orientadas a generar conciencia en la población de los síntomas y riesgos de la tuberculosis, y estrategias de abogacía para lograr el compromiso político de las autoridades nacionales.

- Establecer coordinaciones con otros actores claves para el control de la tuberculosis, como ministerios de justicia, finanzas, y otros socios técnicos.

- Realizar una investigación operacional multicéntrica en relación al tratamiento de la tuberculosis latente en los PBP.

Además, la oficina regional para el control de la tuberculosis de OPS se comprometió a realizar acciones de abogacía con las autoridades nacio- 
nales de salud para reforzar el compromiso político necesario para avanzar en la eliminación de la tuberculosis en los países de baja prevalencia.

\section{Bibliografía}

1.- GONZÁLEZ E, ARMAS L. Eliminación de la tuberculosis como problema de salud pública: una elección acertada. Rev Esp Salud Pública 2007; 81: 59-62.

2.- OFICINA REGIONAL PARA EL CONTROL DE LA TUBERCULOSIS EN LAS AMÉRICAS. Agenda de la VI Reunión Regional de Países de Baja prevalencia de Tuberculosis en Las Américas. Organización Panamericana de la Salud. Santiago de Chile 2013.
3.- DEL GRANADO M. Objetivos y resultados esperados de la reunión. Santiago de Chile, 2013.

4.- DEL GRANADO M. Control de la tuberculosis en los países de baja prevalencia. Santiago de Chile 2013.

5.- RODRÍGUEZ J. Presentación de Uruguay como país de baja prevalencia. Santiago de Chile 2013.

6.- MATA Z. Presentación de Costa Rica como país de baja prevalencia. Santiago de Chile, 2013.

7.- LLANES M J. Presentación de Cuba como país de baja prevalencia. Santiago de Chile 2013.

8.- HERRERA T. Presentación de Chile como país de baja prevalencia. Santiago de Chile 2013.

9.- DEL GRANADO M. Conclusiones y recomendaciones de la VI Reunión de países de baja prevalencia. Santiago de Chile 2013.

\title{
Comentarios sobre los países de baja prevalencia de tuberculosis en América Latina
}

\author{
ÁLVARO YÁÑEZ DEL V.*
}

\author{
Commentaries on the epidemiological situation of Latin American countries \\ of low prevalence of tuberculosis
}

\section{Situación}

Chile, Costa Rica, Cuba y Uruguay, han logrado una reducción de la endemia tuberculosa a tasas inferiores a 20 casos por 100.000 habitantes, tasa que corresponde al umbral de eliminación de la tuberculosis como problema de Salud Pública, condición epidemiológica que se consideró posible alcanzar y que abría la posibilidad cierta de lograr la eliminación de la enfermedad en el largo plazo. Actualmente se considera que la enfermedad se ha eliminado de una comunidad, cuando la incidencia llega a 1 caso o menos por 1.000.000 habitantes.

Si bien con las tasas actuales la mayor parte de la población en estos países tiene un riesgo muy bajo de ser infectada y enfermar, en todos ellos existen grupos más o menos circunscritos, con un riesgo varias veces superior al del promedio nacional, constituyendo estos sectores vulnerables, junto con la población mayor de 60 años, el último reducto de la enfermedad. A lo anterior se suma en estos países, en los últimos años, la detención del proceso de reducción de la incidencia, lo cual determina un exceso de morbilidad en relación a lo esperado de haberse mantenido la tendencia observada en la última década.

Esta es una poderosa señal de alarma, que pone en evidencia que se mantiene la cadena de transmisión de la enfermedad, no continúa la reducción de la incidencia de la morbilidad y se mantiene la mortalidad, sin descender a su mínima expresión.

\section{Amenaza}

En caso de mantenerse esta situación por un tiempo superior a 4 ó 5 años, se puede comenzar a asistir a la reversión epidemiológica de la endemia de la tuberculosis, con ascenso de la incidencia, aumento de la prevalencia, incremento del número de fracasos, mortalidad por la enfermedad y surgimiento de casos tuberculosis

* Asesor Programa de Control de la Tuberculosis, Ministerio de Salud, Chile y ex Asesor Regional de Tuberculosis de la Organización Panamericana de la Salud. 